\title{
Distrofia retiniana com onda rápida escotópica (DRORE) associada à síndrome dos cabelos anágenos frouxos (SCAF). Parte I: Achados oftalmológicos
}

\author{
Scotopic fast wave retinal dystrophy(SFWRD) associated with looseanagen hair syndrome \\ (LAHS). Part I:Ophthalmological findings
}

\author{
Mário Teruo Sato ${ }^{1}$ \\ Rodrigo Marzagãó ${ }^{2}$ \\ Carlos Augusto Moreira Júnior ${ }^{3}$
}

Universidade Federal do Paraná - Disciplina de Oftalmologia, Centro da Visão, Hospital de Clínicas. R. Pasteur, 26 - Curitiba (PR) CEP 80250-080

${ }^{1}$ Professor Convidado e Responsável pelo Serviço de Neuro-Oftalmologia e Eletrofisiologia Ocular da Disciplina de Oftalmologia da Universidade Federal do Paraná.

${ }^{2}$ Médico formado pela Universidade Federal do Paraná. ${ }^{3}$ Reitor da Universidade Federal do Paraná, Professor Titular da Disciplina de Oftalmologia da Universidade Federal do Paraná.

Endereço para correspondência: Dr. Mário Teruo Sato - Av. Winston Churchill, 1323 - Curitiba (PR) CEP 81130-000

E-mail: mariots@super.com.br

Recebido para publicação em 20.11.2003

Versão revisada recebida em 23.03.2004 Aprovação em 30.03.2004

Nota Editorial: Pela análise deste trabalho e por sua anuência na divulgação desta nota, agradecemos ao Dr. André Barbosa Castelo Branco.

\begin{tabular}{|c|}
\hline RESUMO \\
\hline $\begin{array}{l}\text { Objetivos: Descrever os achados oftalmológicos de nova distrofia macular } \\
\text { associada à síndrome dos cabelos anágenos frouxos (SCAF). Métodos: } \\
\text { Uma família de onze pacientes, quatro deles afetados ocularmente, foram } \\
\text { examinados. Sete pacientes foram submetidos aos seguintes exames: } \\
\text { avaliação oftalmológica completa, teste de cores, ecografia, angiografia, } \\
\text { campo visual, topografia de papila, eletro-oculograma (EOG), eletrorreti- } \\
\text { nograma (ERG), testes laboratoriais e exame dermatológico, teste de suor, } \\
\text { microscopia óptica(MO) e microscopia eletrônica de varredura (MEV) dos } \\
\text { fios de cabelo. Em quatro pacientes, filhos dos afetados, realizamos somente } \\
\text { exame oftalmológico, microscopia óptica e microscopia eletrônica de } \\
\text { varredura dos fios de cabelo. Resultados: Dos quatro pacientes afetados, } \\
\text { em duas irmãs encontramos no fundo de olho dispersões pigmentares em } \\
\text { pólo posterior da retina, com coloboma macular. Em dois de seus irmãos } \\
\text { foram encontradas as mesmas alterações oculares, com maior pigmentação } \\
\text { e coloração amarelada em área macular e ausência de coloboma. Os } \\
\text { resultados do eletro-oculograma e eletrorretinograma estavam dentro dos } \\
\text { limites de normalidade em três pacientes semalterações oculares; por outro } \\
\text { lado,o eletro-oculogramafoi subnormaleoeletrorretinogramafoi subnormal } \\
\text { com tempo de culminação diminuído (rápido) na fase escotópica dos } \\
\text { pacientes com a distrofia macular, sugerindo que a alteração dessa distrofia } \\
\text { se encontra em nível do epitélio pigmentado da retina. Devido a esse } \\
\text { comportamento peculiar do tempo de culminação, este achado pode ser } \\
\text { considerado como fundamental para o diagnóstico desta distrofia. A } \\
\text { denominação sugerida é de distrofia retiniana com onda rápida escotópica } \\
\text { (DRORE). A microscopia óptica e microscopia eletrônica de varredura dos } \\
\text { fios de cabelo confirmaram a síndrome dos cabelos anágenos frouxos. } \\
\text { Conclusões: Descrevemos os achados da distrofia retiniana com onda } \\
\text { rápida escotópica associada à síndrome dos cabelos anágenos frouxos, } \\
\text { distrofia cujos achados fundoscópicos são diferentes entre homens e } \\
\text { mulheres e com traçado eletrorretinográfico característico. }\end{array}$ \\
\hline
\end{tabular}

Descritores: Degeneração macular; Doenças do cabelo; Síndrome cabelo; Coloboma; Microscopia eletrônica de varredura; Microscopia eletrônica; Eletrorretinografia 


\section{INTRODUÇÃO}

Distrofias maculares são degenerações progressivas da retina e/ou coróide que afetam predominantemente a área macular e não apresentam etiologia definida. Essas alterações tornam-se manifestas na infância como um distúrbio determinado geneticamente, provavelmente na função das enzimas e metabolismo. Isto ocorre em forma de base displásica, em que células normais ou tecidos gradualmente deterioram-se e morrem prematuramente. As distrofias maculares são caracterizadas pelo seguinte elenco: 1) alterações predominantemente no pólo posterior; 2) de caráter familiar, bilaterais e frequientemente de envolvimento simétrico; 3 ) associadas com diminuição da acuidade visual; 4) tem início em idade precoce; 5) progressão lenta e 6) geralmente sem alterações físicas gerais ou anormalidades laboratoriais ${ }^{(1)}$.

Síndrome dos cabelos anágenos frouxos (SCAF) é um distúrbio que se apresenta precocemente na infância e caracteriza-se por alopécia difusa ou localizada resultante de cabelos fracamente ancorados na fase anágena (fase de crescimento ativo dos cabelos). Esses cabelos são facilmente retirados do couro cabeludo e de forma indolor ${ }^{(2)}$. A herança é autossômica dominante, e os achados essenciais são: 1) cabelos facilmente retirados do couro cabeludo quando puxados; 2) encontramse na fase anágena e 3) há ausência das bainhas radiculares externa e interna ${ }^{(3)}$. Os achados característicos na microscopia eletrônica de varredura (MEV) são a presença de formações canaliculares que ocorrem ao longo do folículo piloso e enrugamento da cutícula próxima à raiz ${ }^{(2)}$.
Neste trabalho, descrevemos uma família com onze indivíduos; destes, quatro (dois irmãos e duas irmãs) tinham o achado incomum de distrofia macular e a SCAF, dois apresentavam somente a SCAF, sendo considerados como heterozigotos. A determinação dos locais das alterações na retina desta distrofia macular pela angiografia, eletro-oculograna (EOG) e principalmente o papel fundamental do eletrorretinograma (ERG) para a caracterização desta distrofia são descritas.

\section{MÉTODOS}

Foram examinados 11 pacientes, sendo que em 7 deles (linha pontilhada do heredograma na figura 1, casos II-19, III20-25) foram submetidos ao exame oftalmológico completo: 1) acuidade visual (AV), motilidade ocular, pressão ocular (Po), biomicroscopia do vítreo e fundo de olho sob dilatação; 2) teste de cores (Teste de Ishihara e Farnsworth D-15); 3) ecografia ocular; 4) campo visual (CV) (Humphrey Instruments); 5) Topografia de disco óptico (TOPPS-Laser Diagnostic Technologies, Inc., San Diego, CA); 6) angiografia (retinógrafo TOPCON-Imagenet 50IA); 6) eletrorretinograma (ERG) e eletro-oculograma (EOG) (EPIC-2000 LKC Technologies, Inc., Gaithersburg, USA).

O EOG foi realizado no aparelho de eletrofisiologia ocular (EPIC-2000). De acordo com a nossa normatização, foram considerados de EOG alterado os valores da relação de Arden menores que $2,097^{(4)}$.

O ERG foi realizado no aparelho de eletrofisiologia ocular (EPIC-2000); onde foram obtidos valores de normalidade para um intervalo de confiança de $95 \%{ }^{(5)}$.

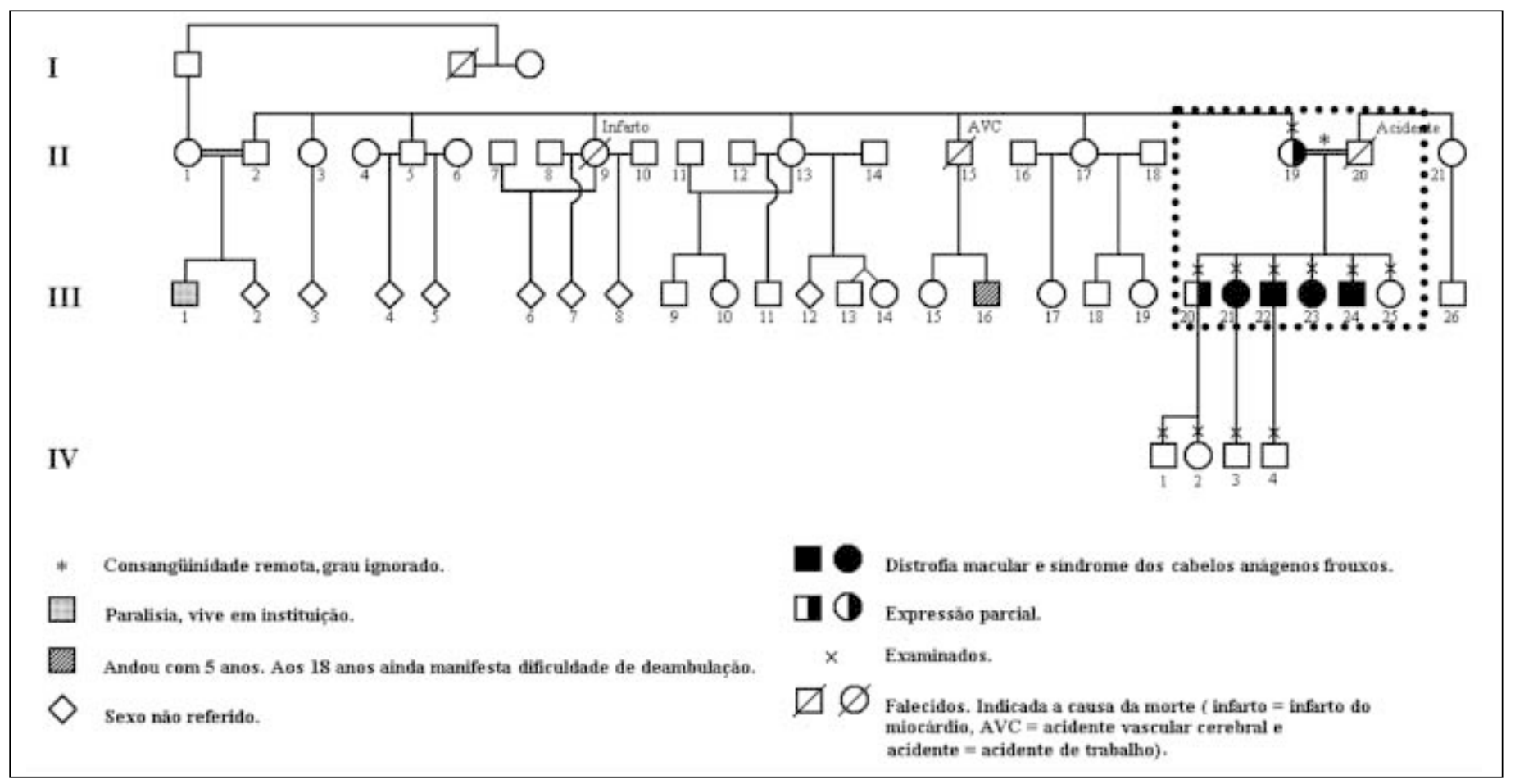

Figura 1 - Heredograma da família 
$\mathrm{Na}$ avaliação clínica foram levantados os seguintes exames:

- Exame dermatológico completo.

- Testes laboratoriais (hemograma completo, provas de função hepática, bioquímica sangüínea, sorologia para toxoplasmose, sorologia para Lues - VDRL e FTA-Abs, parcial de urina e parasitológico de fezes).

- Radiografia panorâmica dos dentes.

- Teste de suor.

- Tricograma.

Microscopia óptica (MO) e MEV dos fios de cabelos (metalização com ouro e observação no aparelho de MEVPhilips SEM 505).

Em 4 pacientes (Figura 1, casos IV 1-4) descendentes dos pacientes afetados, foram examinados para verificar se apresentavam alterações oculares ou dermatológicas. Nesses pacientes, foram realizados os seguintes exames: 1) AV, motilidade ocular, biomicroscopia do vítreo e fundo de olho sob dilatação; 2) teste de cores (Ishihara e Farnsworth D-15); 3) tricograma (não realizado no caso IV-1).

\section{RESULTADOS}

Descrevemos uma família de cor negra, proveniente de Paranaguá (PR). Os resultados da anamnese, exame oftalmológico e complementares (teste de cores, retinografia, angiografia e ecografia) já foram descritos ${ }^{(6)}$. A avaliação dermatológica - exame clínico, tricograma, MO e MEV dos fios de cabelo, confirmaram o diagnóstico de SCAF (resumo na tabela $1 \mathrm{e}^{(4)}$ ).

A história clínica, e posterior investigação do cabelo, determinaram o diagnóstico da distrofia, mostrando tratar-se de uma associação incomum com a SCAF. As radiografias dos dentes, exame das unhas, teste de suor e partes do exame oftalmológico (motilidade oculares, pressão ocular (Po) e biomicroscopia do vítreo) foram normais em todos os pacientes examinados na linha pontilhada do heredograma da figura 1 . Os exames laboratoriais (hemograma completo, provas de fun- ção hepática, bioquímica sangüínea, sorologia para Lues, parcial de urina e parasitológico de fezes) foram normais, exceto o paciente III-24, que apresentou hipotireoidismo. Sorologia para toxoplasmose com IgM não reagente em todos os pacientes, IgG reagente nos seguintes pacientes (casos III-25, III-20, III-22, III-23 e III-24) e não reagente nos pacientes (casos II-19 e III-21).

Nos filhos dos afetados (figura 1, casos IV 1-4), o exame oftalmológico foi normal e aparentemente não houve comprometimento do cabelo.

Os resultados de todos os exames oftalmológicos, exceto de eletrofisiologia ocular, estão resumidos na tabela 2. De maneira geral, nos indivíduos afetados (dois irmãos e duas irmãs, probandos, casos III-21 a III-24) apresentaram alterações confinadas ao pólo posterior da retina, principalmente na mácula, associada à história da deficiência do crescimento dos cabelos. As alterações evidenciadas incluem distrofia macular, cuja característica fundoscópica é a diferença entre os irmãos: nas duas irmãs, há distrofia macular associada a coloboma macular; nos irmãos, há a distrofia macular com aumento de pigmentação amarelada na mácula (Figura 2) e ausência de coloboma macular, achados confirmados pela ecografia. No teste de cores, o defeito mais comum foi o deutan nas mulheres e tritan nos homens. Quanto ao CV, houve principalmente alteração do CV central e preservação do CV periférico. As mulheres mostraram pior acuidade visual que nos homens.

Os resultados do EOG e ERG encontram-se na tabela 3. Nota-se a maioria dos parâmetros normais do ERG e EOG nos paciente não afetados ocularmente (Tabela 3). Nos pacientes afetados ocularmente, os achados eletrorretinográficos foram: 1) diminuição da amplitude e 2) diminuição do tempo de culminação na fase escotópica. Estes podem ser considerados característicos da distrofia descrita, sendo semelhantes entre os irmãos e irmãs (Figuras 3A, 3B). O potencial oscilatório encontrou-se diminuído em dois dos pacientes afetados (Figura 4 e Tabela 3, caso III-23 e III-24). Na fase fotópica, houve diminuição da amplitude com tempo de culminação normal ou

\begin{tabular}{|c|c|c|c|c|c|c|c|c|c|}
\hline \multirow[b]{2}{*}{ Heredograma } & \multirow[b]{2}{*}{ Sexo } & \multirow[b]{2}{*}{$\begin{array}{l}\text { Idade } \\
\text { (anos) }\end{array}$} & \multicolumn{2}{|c|}{ Tricograma (médias) } & \multirow[b]{2}{*}{$\begin{array}{c}\text { Anágenos } \\
\text { distróficos (MO) }\end{array}$} & \multirow[b]{2}{*}{$\begin{array}{c}\text { Queda } \\
\text { espontânea (MO) }\end{array}$} & \multirow[b]{2}{*}{$\begin{array}{l}\text { Cabelo } \\
\text { (clínica) }\end{array}$} & \multirow[b]{2}{*}{$\begin{array}{c}\text { Haste do } \\
\text { cabelo (MEV) }\end{array}$} & \multirow[b]{2}{*}{$\begin{array}{l}\text { Distrofia } \\
\text { macular }\end{array}$} \\
\hline & & & $\begin{array}{l}\text { Anágenos } \\
(\%)\end{array}$ & $\begin{array}{c}\text { Telógenos } \\
(\%)\end{array}$ & & & & & \\
\hline III-25- Caçula, paciente normal & $\mathrm{F}$ & 26 & 87,3 & 12,7 & Ausente & Telógenos & Normal & Normal & Ausente \\
\hline II-19 - Mãe dos pacientes, heterozigota & $\mathrm{F}$ & 60 & 64,6 & 35,4 & Presente & Anágenos & Normal & Alterada & Ausente \\
\hline III-20- Primogênito, heterozigoto & M & 36 & 68,8 & 31,2 & Ausente & Anágenos & Normal & Alterada & Ausente \\
\hline $\begin{array}{l}\text { III-21 - Irmã mais velha afetada } \\
\text { ocularmente, homozigota }\end{array}$ & $\mathrm{F}$ & 34 & 87,9 & 12,1 & Presente & $\begin{array}{l}\text { Anágenos } \\
\text { distróficos }\end{array}$ & $\begin{array}{c}\text { Queda fácil } \\
\text { desde a infância }\end{array}$ & Alterada & Presente \\
\hline $\begin{array}{l}\text { III-22- Irmão mais velho afetado } \\
\text { ocularmente, homozigoto }\end{array}$ & M & 32 & 52,9 & 47,1 & Presente & Telógenos & $\begin{array}{c}\text { Queda fácil } \\
\text { desde a infância }\end{array}$ & Alterada & Presente \\
\hline $\begin{array}{l}\text { III-23- Irmã mais nova afetada } \\
\text { ocularmente, homozigota }\end{array}$ & $\mathrm{F}$ & 30 & 20,9 & 79,1 & Ausente & Anágenos & $\begin{array}{c}\text { Quedafácil } \\
\text { desde a infância }\end{array}$ & Alterada & Presente \\
\hline $\begin{array}{l}\text { III-24- Irmão mais novo afetado } \\
\text { ocularmente, homozigoto }\end{array}$ & M & 28 & 64,4 & 35,6 & Ausente & Anágenos & $\begin{array}{c}\text { Queda fácil } \\
\text { desde a infância }\end{array}$ & Alterada & Presente \\
\hline IV-1 - Filho do paciente III-20, normal & M & 17 & NR & NR & NR & NR & Normal & NR & Ausente \\
\hline IV-2 - Filha do paciente III-20, normal & $\mathrm{F}$ & 12 & 87,4 & 12,6 & Ausente & Telógenos & Normal & NR & Ausente \\
\hline IV-3 - Filha da paciente III-21, normal & M & 9 & 88,3 & 11,7 & NR & NR & Normal & NR & Ausente \\
\hline IV-4 - Filho do paciente III-22, normal & M & 6 & 75,0 & 25,0 & NR & NR & Normal & NR & Ausente \\
\hline
\end{tabular}




\begin{tabular}{|c|c|c|c|c|c|c|c|c|c|c|c|c|c|}
\hline $\begin{array}{l}\text { Heredo- } \\
\text { grama }\end{array}$ & $\begin{array}{l}\text { Sexo/ } \\
\text { idade }\end{array}$ & $\begin{array}{l}\text { Apare- } \\
\text { cimento dos } \\
\text { sintomas }\end{array}$ & $\begin{array}{c}\text { Dificul- } \\
\text { dade de } \\
\text { visãoànoite }\end{array}$ & $\begin{array}{l}\text { Foto- } \\
\text { fobia }\end{array}$ & $\begin{array}{l}\text { AVcc } \\
\text { OD } \\
O E\end{array}$ & $\begin{array}{l}\text { Refração } \\
\text { OD } \\
\text { OE }\end{array}$ & $\begin{array}{l}\text { Ishihara } \\
\text { OD } \\
\text { OE }\end{array}$ & $\begin{array}{l}\text { D-15 } \\
\text { OD } \\
\text { OE }\end{array}$ & $\begin{array}{l}\text { FO } \\
\text { OD } \\
\text { OE }\end{array}$ & $\begin{array}{l}\text { Angiografia } \\
\text { OD } \\
\text { OE }\end{array}$ & $\begin{array}{l}\text { Ecografia } \\
\text { OD } \\
O E\end{array}$ & $\begin{array}{l}\text { CV } \\
\text { OD } \\
O E\end{array}$ & $\begin{array}{l}\text { TOPSS } \\
\text { OD } \\
\text { OE }\end{array}$ \\
\hline $111-25$ & F/26 & - & - & - & $\begin{array}{l}20120 \\
2020\end{array}$ & $\begin{array}{l}\text { Plano } \\
\text { Plano }\end{array}$ & $N_{N}^{N}$ & $N_{N}^{N}$ & $\begin{array}{l}n \\
N\end{array}$ & $\begin{array}{l}\mathrm{N} \\
\mathrm{N}\end{array}$ & $\begin{array}{l}n \\
N\end{array}$ & $\begin{array}{l}\mathrm{N} \\
\mathrm{N}\end{array}$ & $\begin{array}{l}\text { Normal em OD e redução do } \\
\text { diâmetrodo disco ópticoeme }\end{array}$ \\
\hline II-19 & $F / 60$ & - & - & - & $\begin{array}{l}20120 \\
20220\end{array}$ & $\begin{array}{c}\text { Plano AO } \\
\text { Adição+2,50esf. }\end{array}$ & $\begin{array}{l}\mathrm{N} \\
\mathrm{N}\end{array}$ & $\begin{array}{l}\mathrm{I} \\
\mathrm{D}\end{array}$ & $\begin{array}{l}\mathrm{N} \\
\mathrm{N}\end{array}$ & $\begin{array}{l}\mathrm{N} \\
\mathrm{N}\end{array}$ & $\begin{array}{l}\mathrm{N} \\
\mathrm{N}\end{array}$ & $\begin{array}{l}\text { Retraçãodo CV superior } \\
\text { deOD ereduçãodasensibi- } \\
\text { lidadetemporalem OE. }\end{array}$ & $\begin{array}{l}\text { Diminuição darima neural } \\
\text { eaumentodaescavação } \\
\text { em OD. Normal em OE }\end{array}$ \\
\hline III-20 & M36 & - & - & - & $\begin{array}{l}20 / 20 \\
20 / 20\end{array}$ & $\begin{array}{l}\text { Plano } \\
\text { Plano }\end{array}$ & $\begin{array}{l}N \\
N\end{array}$ & $\stackrel{N}{N}$ & $\begin{array}{l}\mathrm{N} \\
\mathrm{N}\end{array}$ & $\begin{array}{l}\mathrm{N} \\
\mathrm{N}\end{array}$ & $\begin{array}{l}\mathrm{N} \\
\mathrm{N}\end{array}$ & $\begin{array}{l}\mathrm{N} \\
\mathrm{N}\end{array}$ & $\begin{array}{l}\mathrm{N} \\
\mathrm{N}\end{array}$ \\
\hline III-21 & F/34 & $\begin{array}{l}\text { Por volta } \\
\text { de11anos }\end{array}$ & Não & Sim & $\begin{array}{l}C D \pm 2 m \\
C D \pm 2 m\end{array}$ & $\begin{array}{c}-0,75 \text { esf. }-1,00 \times 45^{\circ} \\
-0,75 \text { esf. }\end{array}$ & $\begin{array}{l}\text { A } \\
\text { A }\end{array}$ & $\begin{array}{l}D \\
D\end{array}$ & Vide figura2 & $\begin{array}{l}\text { Atrofiadacoriocapilar } \\
\text { edo EPRem pólo posteriore } \\
\text { coloboma macular em AO }\end{array}$ & $\begin{array}{l}\text { Coloboma } \\
\text { macular } \\
\text { em AO }\end{array}$ & $\begin{array}{l}\text { PerdadeCV } \\
\text { inferior em AO }\end{array}$ & $\begin{array}{c}\text { Disco óptico com } \\
\text { diâmetropequenoe } \\
\text { escavaçăonormalem AO }\end{array}$ \\
\hline ||1|-22 & M32 & $\begin{array}{l}\text { Por volta } \\
\text { de7anos }\end{array}$ & Sim & Sim & $\begin{array}{l}20 / 200 \\
20 / 200\end{array}$ & $\begin{array}{l}+1,00 \text { esf. }-1,50 \times 90^{\circ} \\
-2,00 \times 90^{\circ}\end{array}$ & $\begin{array}{l}\text { A } \\
\text { A }\end{array}$ & $\begin{array}{l}\mathrm{T} \\
\mathrm{D}\end{array}$ & Vide figura2 & $\begin{array}{l}\text { Atrofiadacoriocapilar } \\
\text { edo EPRempólo } \\
\text { posterior em AO }\end{array}$ & $\begin{array}{l}\mathrm{N} \\
\mathrm{N}\end{array}$ & $\begin{array}{c}\text { Remanescentede } \\
\mathrm{CV} \text { nasal periférico em AO }\end{array}$ & $\begin{array}{l}\mathrm{N} \\
\mathrm{N}\end{array}$ \\
\hline |II-23 & $\mathrm{F} / 30$ & $\begin{array}{l}\text { Por volta } \\
\text { de7anos }\end{array}$ & Não & Sim & $\begin{array}{l}20 / 200 \\
\mathrm{CD} \pm 2 \mathrm{~m}\end{array}$ & $\begin{array}{l}-0,75 \text { esf. }-1,00 \times 60^{\circ} \\
-1,00 \text { esf. }\end{array}$ & $\begin{array}{l}\text { A } \\
\text { A }\end{array}$ & $\begin{array}{l}\mathrm{T} \\
\mathrm{D}\end{array}$ & Vide figura2 & $\begin{array}{c}\text { Idem ao da irmã mais } \\
\text { velha (caso III-21) em AO }\end{array}$ & $\begin{array}{l}\text { Idem ao dairmã } \\
\text { mais velha } \\
\text { (casolll-21) em AO }\end{array}$ & NR & $\begin{array}{l}\mathrm{N} \\
\mathrm{N}\end{array}$ \\
\hline III-24 & M28 & $\begin{array}{l}\text { Por volta } \\
\text { de7anos }\end{array}$ & Sim & Sim & $\begin{array}{c}20 / 25 \\
20 / 200\end{array}$ & $\begin{array}{r}+2,50 \text { esf. }-2,00 \times 15^{\circ} \\
+2,50 \text { esf. }-1,00 \times 180^{\circ}\end{array}$ & $\begin{array}{l}A \\
A\end{array}$ & $\begin{array}{l}\mathrm{T} \\
\mathrm{T}\end{array}$ & Vide figura2 & $\begin{array}{l}\text { Idem ao do irmão mais } \\
\text { velho (caso III-22) em AO }\end{array}$ & $\begin{array}{l}\mathrm{N} \\
\mathrm{N}\end{array}$ & $\begin{array}{l}\text { Remanes centedeCV } \\
\text { central e periférico em AO }\end{array}$ & $\begin{array}{l}\mathrm{N} \\
\mathrm{N}\end{array}$ \\
\hline $\mathrm{N}-1$ & M17 & - & - & - & $\begin{array}{l}20120 \\
20 / 20\end{array}$ & NR & $\begin{array}{l}\mathrm{N} \\
\mathrm{N}\end{array}$ & $\begin{array}{l}\mathrm{N} \\
\mathrm{N}\end{array}$ & $\begin{array}{l}N \\
N\end{array}$ & NR & NR & NR & NR \\
\hline IV-2 & $F / 12$ & - & - & - & $\begin{array}{l}20120 \\
20220\end{array}$ & $N R$ & $\begin{array}{l}\mathrm{N} \\
\mathrm{N}\end{array}$ & $\begin{array}{l}\mathrm{N} \\
\mathrm{N}\end{array}$ & $\begin{array}{l}\mathrm{N} \\
\mathrm{N}\end{array}$ & NR & $N R$ & NR & NR \\
\hline IV-3 & M/9 & - & - & - & $\begin{array}{l}2020 \\
20220\end{array}$ & NR & $\begin{array}{l}\mathrm{N} \\
\mathrm{N}\end{array}$ & $\begin{array}{l}\mathrm{N} \\
\mathrm{N}\end{array}$ & $\begin{array}{l}\mathrm{N} \\
\mathrm{N}\end{array}$ & NR & NR & NR & NR \\
\hline IV-4 & $\mathrm{M} / 6$ & - & - & - & $\begin{array}{l}20120 \\
20120\end{array}$ & NR & $\begin{array}{l}\mathrm{N} \\
\mathrm{N}\end{array}$ & $\begin{array}{l}\mathrm{N} \\
\mathrm{N}\end{array}$ & $\begin{array}{l}\mathrm{N} \\
\mathrm{N}\end{array}$ & NR & NR & NR & NR \\
\hline
\end{tabular}

\begin{tabular}{|c|c|c|c|c|c|c|c|c|c|c|c|c|c|c|}
\hline \multirow{4}{*}{$\begin{array}{l}\text { Heredo- } \\
\text { grama }\end{array}$} & \multirow{4}{*}{$\begin{array}{l}\text { Sexo/ } \\
\text { idade }\end{array}$} & \multirow{4}{*}{$\begin{array}{l}\text { EOG } \\
\text { OD } \\
\text { OE }\end{array}$} & \multicolumn{2}{|r|}{ E24 } & \multicolumn{2}{|r|}{ EOA } & \multicolumn{2}{|r|}{ EOB } & \multicolumn{2}{|r|}{ PO } & \multicolumn{2}{|r|}{ FO } & \multicolumn{2}{|r|}{ FL } \\
\hline & & & A & LN & $\mathbf{A}$ & LN & $\mathbf{A}$ & LN & $\mathbf{A}$ & LN & $\mathbf{A}$ & LN & $\mathbf{A}$ & LN \\
\hline & & & TC & A & TC & A & TC & A & & A & TC & A & TC & A \\
\hline & & & & TC & & TC & & TC & & & & TC & & TC \\
\hline \multirow[t]{2}{*}{ III-25 } & $F / 26$ & $2,10 \mathrm{~N}$ & $281,71 \mathrm{~N}$ & $229^{1}-326,91^{2}$ & $317,07 \mathrm{~N}$ & $299,34^{1}-376,26^{2}$ & $265,85 \mathrm{~N}$ & $181,48^{1}-329,52^{2}$ & $312,00 \mathrm{~N}$ & $227,88^{1}-364,50^{2}$ & $119,02 \uparrow$ & $54,01^{1}-103,79^{2}$ & $99,50 \mathrm{~N}$ & $67,73^{1}-105,99^{2}$ \\
\hline & & $2,16 \mathrm{~N}$ & $91,50 \mathrm{~N}$ & $89,59-97,71$ & $16,00 \mathrm{~N}$ & $16,01-17,89$ & $45,00 \mathrm{~N}$ & $45,40-49,70$ & & & $29,00 \mathrm{~N}$ & $29,37-31,13$ & $28,00 \mathrm{~N}$ & $27,55-28,79$ \\
\hline \multirow[t]{2}{*}{ II-19 } & $F / 60$ & $1,90 \downarrow$ & $249,02 \uparrow$ & $186-232,21$ & $273,17 \mathrm{~N}$ & $225,47-309,73$ & $221,95 \mathrm{~N}$ & $162,81-250,99$ & $216,50 \mathrm{~N}$ & $179,30-303,84$ & $62,20 \mathrm{~N}$ & $50,71-81,69$ & $87,80 \mathrm{~N}$ & $45,79-94,73$ \\
\hline & & $1,92 \downarrow$ & $98,50 \mathrm{~N}$ & $93,52-98,68$ & $16,50 \mathrm{~N}$ & $16,35-18,85$ & $48,50 \mathrm{~N}$ & $44,59-51,01$ & & & $30,50 \mathrm{~N}$ & $27,42-30,28$ & $29,00 \mathrm{~N}$ & $19,89-31,13$ \\
\hline \multirow[t]{2}{*}{ |II-20 } & M/36 & $2,36 \mathrm{~N}$ & $275,37 \mathrm{~N}$ & $229-326,91$ & $246,95 \downarrow$ & $299,34-376,26$ & $378,05 \uparrow$ & $181,48-329,52$ & $163,90 \downarrow$ & $277,88-364,50$ & $91,95 \mathrm{~N}$ & $54,01-103,79$ & $76,3 \mathrm{~N}$ & $67,73-105,99$ \\
\hline & & $2,01 \mathrm{~N}$ & $97,50 \mathrm{~N}$ & $89,59-97,71$ & $16,00 \mathrm{~N}$ & $16,01-17,89$ & $48,50 \mathrm{~N}$ & $45,40-49,70$ & & & $29,50 \mathrm{~N}$ & $29,37-31,13$ & $29,50 \uparrow$ & $27,55-28,79$ \\
\hline \multirow[t]{2}{*}{ III-21 } & $F / 34$ & $1,44 \downarrow$ & $150,24 \downarrow$ & $229-326,91$ & $175,00 \downarrow$ & $299,34-376,26$ & $137,80 \downarrow$ & $181,48-329,52$ & $242,50 \mathrm{~N}$ & $227-364,50$ & $85,85 \mathrm{~N}$ & $54,01-103,79$ & $52,90 \downarrow$ & $67,73-105,99$ \\
\hline & & $1,36 \downarrow$ & $86,50 \downarrow$ & $89,59-97,71$ & $15,50 \downarrow$ & $16,01-17,89$ & $44,50 \downarrow$ & $45,40-49,70$ & & & $34,50 \uparrow$ & $29,37-31,13$ & $28,90 \uparrow$ & $27,55-28,79$ \\
\hline \multirow[t]{2}{*}{ |II-22 } & $\mathrm{M} / 32$ & $1,85 \downarrow$ & $208,78 \downarrow$ & $229-326,91$ & $175,00 \downarrow$ & $299,34-376,26$ & $64,02 \downarrow$ & $181,48-329,52$ & $235,00 \mathrm{~N}$ & $227,88-364,50$ & $36,10 \downarrow$ & $54,01-103,79$ & $35,40 \downarrow$ & $67,73-105,99$ \\
\hline & & $1,65 \downarrow$ & $83,00 \downarrow$ & $89,59-97,71$ & $14,50 \downarrow$ & $16,01-17,89$ & $46,00 \mathrm{~N}$ & $45,40-49,70$ & & & $35,00 \uparrow$ & $29,37-31,13$ & $29,60 \uparrow$ & $27,55-28,79$ \\
\hline \multirow[t]{2}{*}{ |II-23 } & $\mathrm{F} / 30$ & $1,83 \downarrow$ & $110,49 \downarrow$ & 229-326,91 & $73,78 \downarrow$ & $299,34-376,26$ & $237,20 \downarrow$ & $181,48-329,52$ & $191,70 \downarrow$ & $227,88-364,50$ & $37,07 \downarrow$ & $54,01-103,79$ & $30,20 \downarrow$ & $67,73-105,99$ \\
\hline & & $1,94 \downarrow$ & $86,00 \downarrow$ & $89,59-97,71$ & $15,00 \downarrow$ & $16,01-17,94$ & $47,50 \mathrm{~N}$ & $45,40-49,76$ & & & $32,00 \uparrow$ & $29,37-31,13$ & $30,00 \uparrow$ & $27,55-28,79$ \\
\hline \multirow[t]{2}{*}{ III-24 } & $\mathrm{M} / 28$ & $1,90 \downarrow$ & $101,46 \downarrow$ & $229-326,91$ & $162,20 \downarrow$ & $299,34-376,26$ & $98,78 \downarrow$ & $181,48-329,52$ & $169,90 \downarrow$ & $227,88-364,50$ & $28,78 \downarrow$ & $54,01-103,79$ & $48,50 \downarrow$ & $67,73-105,99$ \\
\hline & & $1,43 \downarrow$ & $86,00 \downarrow$ & $89,59-97,71$ & $15,00 \downarrow$ & $16,01-17,89$ & $44,00 \downarrow$ & $45,40-49,70$ & & & $27,00 \downarrow$ & $29,37-31,13$ & $27,90 \mathrm{~N}$ & $27,55-28,79$ \\
\hline \multicolumn{15}{|c|}{ 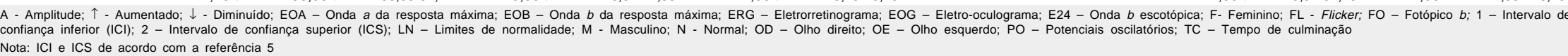 } \\
\hline
\end{tabular}



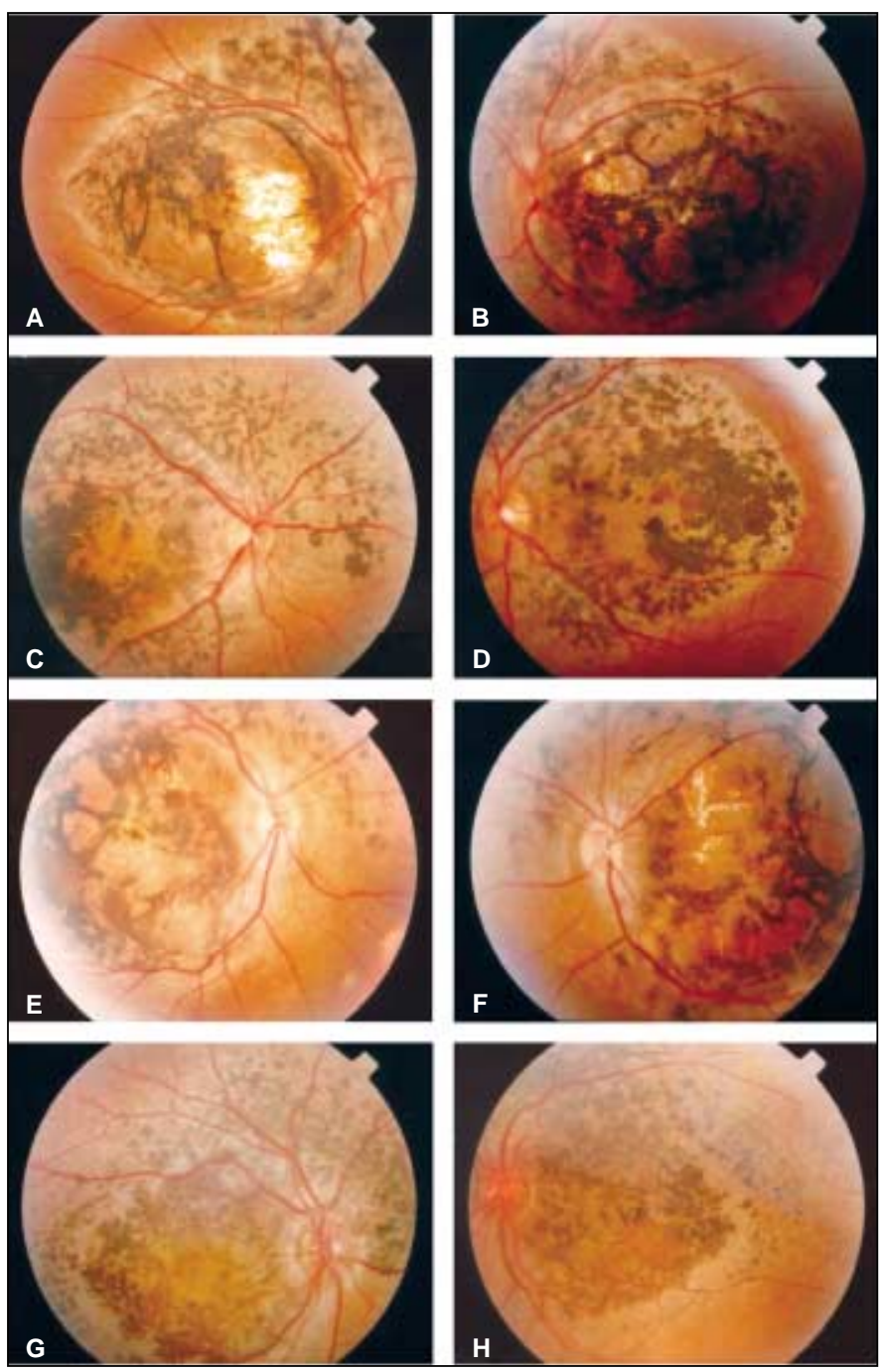

Figura 2 - Retinografia dos pacientes afetados ocularmente. 2A-B: Paciente feminina (caso III-21) - dispersão pigmentar em pólo posterior de tamanhos e formas variados e presença de coloboma em área macular; nota-se pequena área da retina nasal inferior normal; 2C-D: Paciente masculino (caso III-22) - dispersão pigmentar em pólo posterior de tamanho e formas variados e com deposicão de pigmento amarelado em área macular; nota-se pequena área da retina nasal inferior normal; 2E-F: Paciente feminina (caso III-23) - fundo de olho similar ao da irmã (caso III-21); 2G-H: Paciente masculino (caso III-24) - fundo de olho similar ao do irmão (caso III-22)

aumentado (Figuras 5A, 5B). O EOG foi subnormal nos pacientes afetados e normal nos não afetados, exceto no caso II-19 (Tabela 3).

\section{DISCUSSÃO}

O achado fundamental dessa distrofia é o ERG com a onda rápida, ou seja, tempo de culminação diminuído, amplitude diminuída na fase escotópica e fase fotópica com tempo de culminação alargado ou retardado e amplitude diminuída. $\mathrm{O}$ EOG mostrou-se alterado em todos os pacientes afetados ocularmente. Não encontramos relatos similares na literatura em relação a estes achados.
O EOG alterado indica alteração no epitélio pigmentado da retina (EPR). Nas distrofias maculares, quando o ERG é afetado o EOG é também afetado. As alterações do EOG e a presença de dispersões pigmentares no pólo posterior indicam que o defeito primário da distrofia provavelmente localiza-se a nível do EPR. ERG e EOG afetados indicam alteração generalizada da retina na DRORE, como no caso da doença de Stargardt, em que inicialmente o ERG e EOG são normais e posteriormente são afetados. Quando esta se torna difusa, com envolvimento do centro para a periferia, o ERG e o EOG tornam-se anormais ${ }^{(7)}$. Concluindo, a distrofia é macular, porque atinge o pólo posterior, com predominância das lesões na área macular; vasos e disco óptico são normais. A localização primária mais provável da lesão na retina é o EPR, devido ao fato de o EOG ser subnormal e haver distúrbios pigmentares, a exemplo do que ocorre nas degenerações pigmentárias hereditárias da retina. Com a evolução, houve atrofia do EPR com exposição da coriocapilar, como demonstrado pela angiografia e EOG alterados. Uma vez que o ERG é subnormal, a retina neurosensorial provavelmente também é afetada. A onda $a$ subnormal indica alteração nos fotorreceptores, localizados no terço externo da retina e a onda $b$ subnormal, alteração nas células bipolares e de Muller do terço médio da retina. Como exemplo, em pacientes com a distrofia reticular do epitélio pigmentado de Sjögren, o ERG geralmente é normal, provavelmente porque a retina neurosensorial não é envolvida e o EOG é subnormal, devido à alteração no $\mathrm{EPR}^{(8)}$.

A amplitude e o tempo de culminação são achados independentes em um ERG, sendo o tempo de culminação sujeito a menos variações que a amplitude ${ }^{(9)}$. O tempo de culminação reflete com maior sensibilidade a atividade da onda $b$ na camada nuclear interna do que a amplitude. Também são pouco influenciados no curso da adaptação ao escuro. Geralmente, quando há diminuição da amplitude, há o aumento no tempo de culminação, como ocorre nas distrofias ${ }^{(10)}$. O tempo de culminação pode tornar-se rápido quando há o aumento da intensidade do estímulo, para a onda $b^{(11)}$ e para a onda $a^{(12)}$. Outras situações descritas são a onda supernormal com encurtamento do tempo de culminação, no curso da adaptação ao escuro em casos de albinismo universal, ou no albinismo ocular devido ao olho ser translúcido. O tempo de culminação da onda $a$ e $b$ se comporta de maneira diferente no curso de adaptação ao escuro, mas ambas atingem um platô em torno dos 24 minutos de adaptação. O tempo de culminação dos componentes $a 2, b 1$ e $b 2$ aumentam com o decorrer da adaptação; de maneira oposta, o tempo de culminação da onda $a l$ e p2 permanece sem mudanças ${ }^{(13)}$.

$\mathrm{Na}$ metodologia empregada neste trabalho, os pacientes foram adaptados ao escuro de 25 a 30 minutos, tempo suficiente para que a onda do ERG adquira sua amplitude máxima, com estabilização do tempo de culminação. Por estes motivos, o achado de encurtamento do tempo de culminação das ondas (ou onda rápida na fase escotópica - Figura 3A, 3B e Tabela 3), principalmente da onda $a l$ em todos os pacientes afetados ocularmente (nota-se pela Figura 3B que o cursor foi colocado 


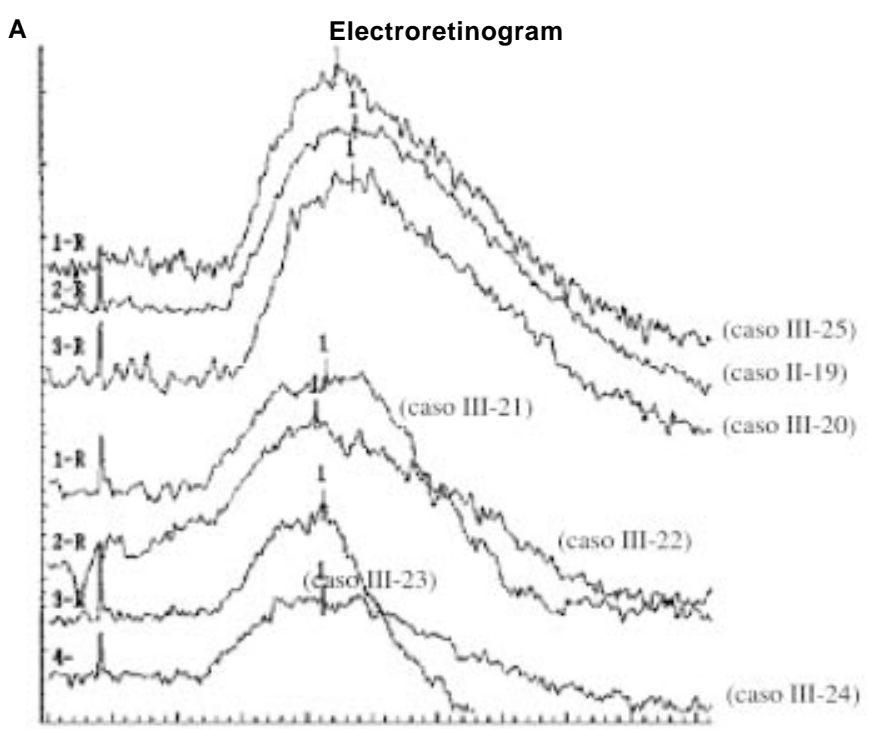

$5 \mathrm{~ms}$ per division

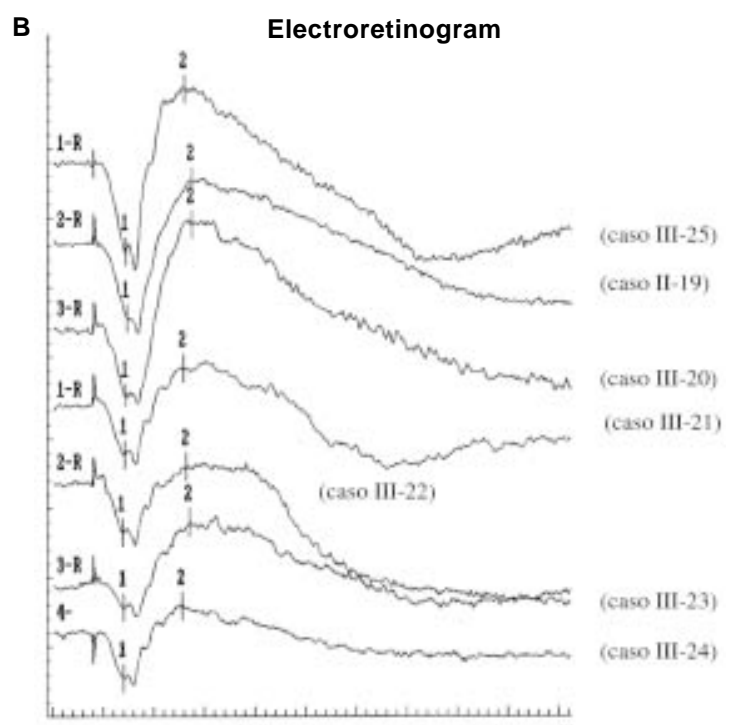

$5 \mathrm{~ms}$ per division

Figura 3 - A - Etapa 1 do ERG - escotópico, flash simples (24 dB), onda b escotópica (E24): cursor no ponto mais alto da onda (1). Resultados da amplitude (A) e tempo de culminação (TC) na tabela 3; B - Etapa 2 do ERG - escotópico, flash simples (0 dB), onda a e b da resposta máxima escotópica (EOA e EOB): cursor da onda $a(1)$ e da onda $b$ (2). Resultados da amplitude (A) e tempo de culminação (TC) na tabela 3

em al para determinar-se a amplitude e o tempo de culminação), podem ser considerado como característico dessa distrofia, já que o tempo de culminação deste componente não varia com a adaptação ao escuro. Desta maneira, sugerimos que o nome desta distrofia seja de distrofia retiniana com onda rápida escotópica ou DRORE. De maneira diferente, a fase fotópica segue o comportamento padrão das distrofias, com diminuição da amplitude e com o aumento (atraso ou alargamento) do tempo de culminação (caso III-21, 22 e 23) ou normal (caso III-24).

Estes achados parecem ser únicos e fundamentais para a caracterização dessa distrofia, devido a pouca variabilidade do tempo de culminação, como já foi demonstrado em trabalhos prévios ${ }^{(9,13)}$. Quanto aos pacientes sem alterações oculares (casos II-19, III-20, III-25), somente a mãe, de 60 anos, apresentou EOG provavelmente normal (relação de Arden de 1,90 no OD e 1,92 no OE); o EOG para esta paciente provavelmente deve ser normal para a sua idade, pois, com o aumento da idade, há o decréscimo do número de células do $\mathrm{EPR}^{(14)}$, e isto deva contribuir para a diminuição da relação de Arden do EOG. Em relação ao ERG, exceto no paciente III-20, encontramos alterações, como a diminuição da amplitude da onda $a$, do potencial oscilatório e aumento do tempo de culminação do flicker. Todas as outras etapas do ERG, principalmente o tempo de culminação da fase escotópica, podem ser consideradas como normais nesse paciente sem alterações oculares. A onda $a$ representa atividade dos fotorreceptores, e o flicker, atividade dos cones (fase fotópica); como não houve alterações nas outras etapas do ERG, e este paciente apresentou exame oftalmológico dentro da normalidade, estes achados podem ser interpretados como variação da normalidade ou manifestação sutil da distrofia, já que este paciente é hete-

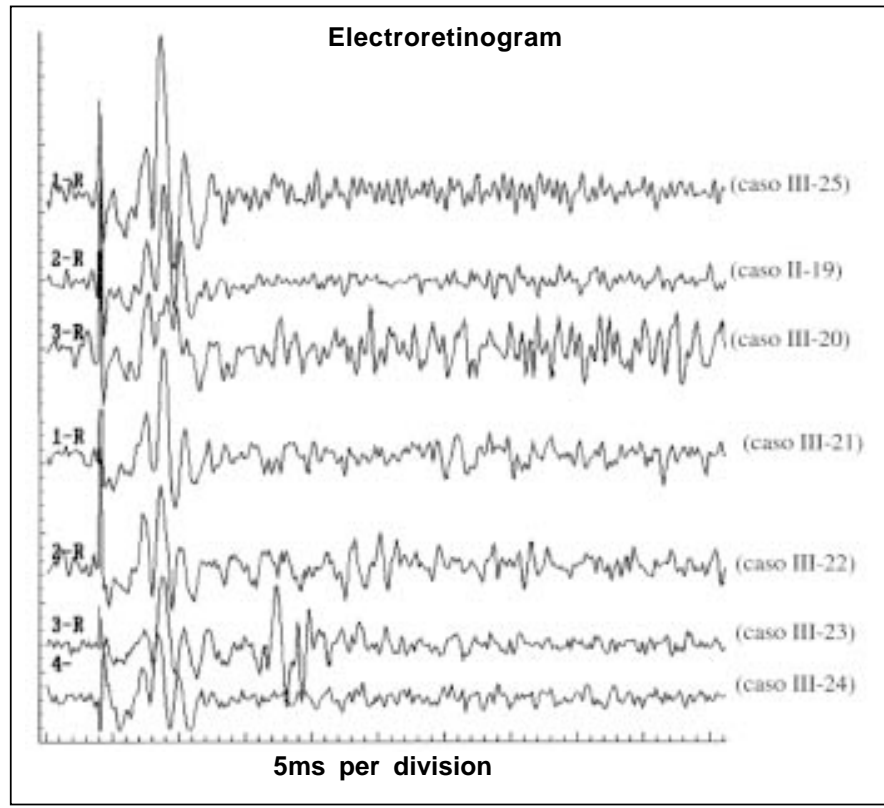

Figura 4 - Etapa 3 do ERG - escotópico, flash simples $(0 \mathrm{~dB})$, potenciais oscilatórios (PO): resultados da amplitude (A) na tabela 3

rozigoto (Tabela 1 e discussão na parte II deste trabalho). Quanto aos potenciais oscilatórios, estes são muito sensíveis às alterações vasculares da retina (diabetes, obstruções venosas e arteriais) e a opacificações dos meios transparentes ${ }^{(5)}$. Investigações clínicas e laboratoriais não evidenciaram quaisquer alterações citadas acima; o exame biomicroscópico e a angiografia normais pressupõem, desta maneira, que o potencial oscilatório do exame não reflete, neste paciente, uma alte- 


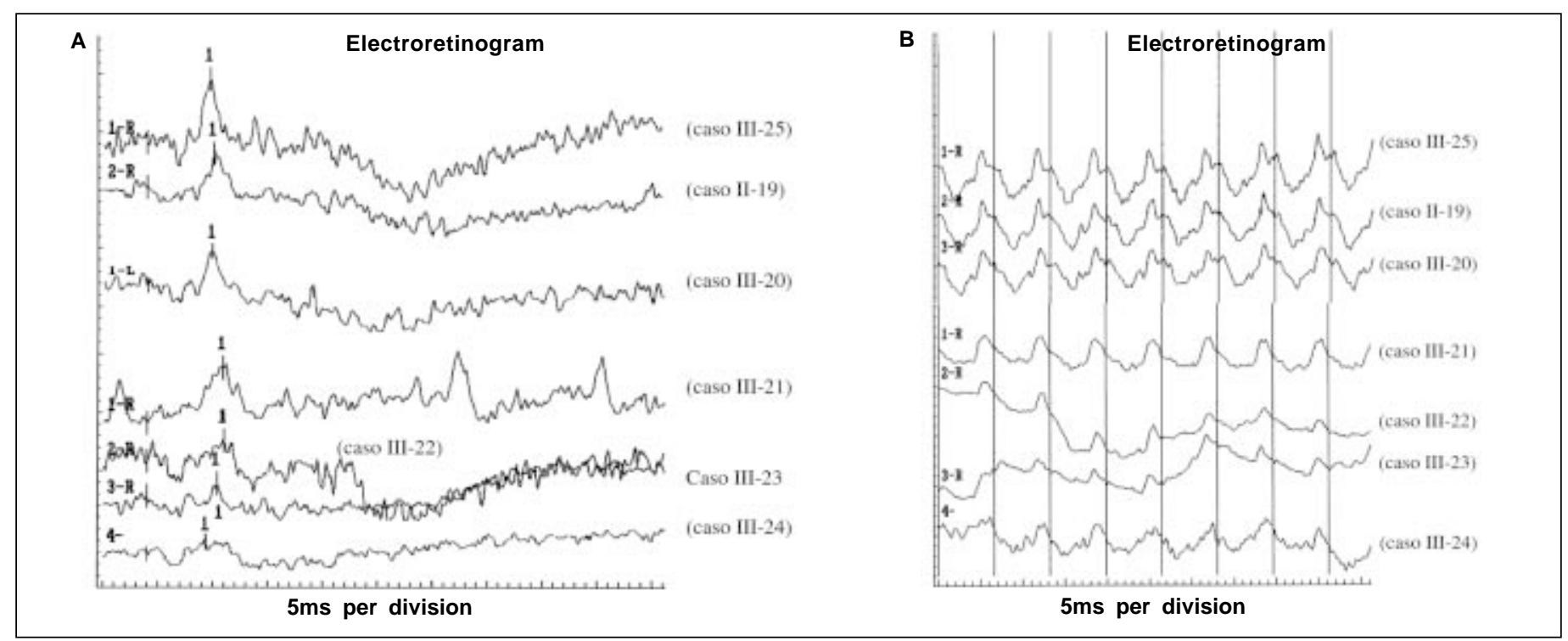

Figura 5 - A - Etapa 4 do ERG - fotópico, flash simples (0 dB), fotópico $b$ (FO): cursor no ponto mais alto da onda (1) resultados da amplitude (A) e tempo de culminação (TC) na tabela 3; B - Etapa 5 do ERG - fotópico, $30 \mathrm{~Hz}$ flicker (FL). Resultados da amplitude (A) e tempo de culminação (TC) na tabela 3

ração patológica, já que as outras etapas do ERG e a investigação clínica e oftalmológica foram normais. Conclui-se que do ponto de vista eletrofisiológico, os pacientes heterozigotos examinados (casos II-19 e III-20) não afetados ocularmente, mas com alterações capilares, não são portadores de alterações a nível da retina, ao contrário de pacientes com alopécia e sem problemas oculares em que se evidenciaram alterações no $\mathrm{EOG}^{(15)}$.

A hipótese inicial para os pacientes afetados (Figura 1 caso III-21, III-22, III-23 e III-24), foi a de retinite pigmentosa inversa, que é caracterizada por uma concentração anormal de pigmentos, envolvendo a mácula, que pode cursar com estreitamento dos vasos retinianos e palidez do disco óptico ${ }^{(16)}$. Pelo fato de os pacientes com a DRORE apresentarem disco óptico normal, vasos retinianos normais e dispersão pigmentar difusa em pólo posterior, descartamos a hipótese diagnóstica de retinite pigmentosa inversa.

A idade de aparecimento dos sintomas nos pacientes com a DRORE na $1^{\underline{a}}$ década de vida (7-11 anos), é compatível com a idade de aparecimento de outras distrofias, tais como a distrofia de cones, na $1^{\underline{a}}-2^{\underline{a}}$ décadas de vida, fundus flavimaculatus de 8 aos 16 anos e doença de Stargardt de 8-16 anos.

Os achados fundoscópicos foram diferentes entre homens e mulheres, com a presença de coloboma macular nas irmãs (evidenciadas pela ecografia e pela refração; pacientes apresentavam miopia) e aumento de pigmentação amarelada na mácula e ausência de coloboma macular nos irmãos (evidenciadas pela ecografia e pela refração; pacientes apresentavam hipermetropia). Esses achados são diferentes de outras distrofias maculares, nas quais a lesão predominante encontra-se na área macular, como a distrofia de $\operatorname{cones}^{(17)}$, doença de Stargardt, distrofia da Carolina do Norte e distrofia central areolar da coróide ${ }^{(7)}$. As dispersões pigmentares em pólo posterior são encontradas na retinite pigmentosa pericentral (in- versa) e central ${ }^{(16)}$ ou na distrofia reticular do epitélio pigmentado da retina de Sjögren, caracterizado por pigmento granular que migra em direção à periferia em formato de rede, que lembra redes de pesca com seus nós.

Coloboma refere-se ao defeito de fechamento da fissura embrionária durante a embriogênese. $\mathrm{O}$ termo coloboma macular também é usado de forma errônea para designar lesões maculares secundárias a infecção intra-uterina (toxoplasmose), não resultante do defeito do fechamento da fissura embrionária. Em alguns casos o estafiloma macular parece-se com o coloboma macular, e ambos os termos são usados como sinônimos $^{(6,18)}$. Exames para lues e toxoplasmose (IgM) negativos, com ausência de exposição à toxoplasmose (IgG não reagente) na mãe dos pacientes afetados (caso II-19) e simetria das lesões maculares em ambos os olhos nas pacientes femininas afetadas (casos III-21 e III-23), descartam a possibilidade de que nessas pacientes o coloboma macular seja causada por infecções intra-uterinas (toxoplasmose ou lues).

A presença de ectasia da esclera nas pacientes femininas com coloboma macular de causa familial associada à causa sistêmica (distúrbio capilar), indicam que a lesão pode ter acontecido precocemente durante o desenvolvimento e provavelmente não relacionado com o fechamento da fissura embrionária. Há somente um relato da associação entre a SCAF e alterações oculares (coloboma envolvendo o disco óptico e a mácula), em um paciente masculino ${ }^{(19)}$. Diferentemente, na DRORE encontrou-se a associação entre a distrofia macular e coloboma macular somente nas pacientes femininas (casos III-21, III-23). Outras associações são descritas com o coloboma ocular; como a amaurose congênita de Leber, distrofia retiniana, atrofia da periferia da retina e displasia coriorretiniana difusa. Não se encontram relatos da associação entre coloboma macular e distrofia macular até o presente momento. 
A fotofobia é queixa freqüente em pacientes que apresentam distrofia de cones, que se caracteriza por apresentar inicialmente em fundo de olho; perda de reflexo foveal e granulações na mácula, evoluindo para lesão em forma de "olho de boi" ${ }^{(7,17)}$. A dificuldade visual à noite é comum nos pacientes que apresentam retinite pigmentosa. Provavelmente a nictalopia nos pacientes masculinos afetados ocularmente (casos III22 e III-24) deve-se à melhor AV. Esses pacientes não apresentavam no fundo de olho e no exame eletrofisiológico características compatíveis com a retinite pigmentosa.

A acuidade visual na maioria das distrofias maculares varia de 20/200 a 20/400 (17) na DRORE associada à SCAF; a AV variou desde $20 / 25$ a 20/200 nos homens e 20/200 a CD a 2 metros nas mulheres, sendo pior nelas provavelmente devido a maior lesão na área macular com a presença da coloboma macular.

Os pacientes afetados com distrofia macular apresentaram alterações no teste para visão de cores. As mulheres (caso III21 e III-23) apresentaram a pior acuidade visual: a tendência do defeito no teste de Farnsworth D-15 foi do tipo deutan. Nos homens, a tendência do defeito foi do tipo protan (caso III-22) e tritan (caso III-24) no irmão com a melhor AV. Esses achados correlacionam-se bem com a AV em pacientes com retinosquisis ligada ao $\mathrm{X}$, em que os defeitos do tipo tritan, quando estão no início da patologia, apresentaram melhor AV; com a progressão da patologia, os pacientes apresentaram o defeito tipo deutan, com a piora da $\mathrm{AV}^{(20)}$. De modo oposto, no fundus flavimaculatus em que os pacientes apresentaram defeito deutan-protan (vermelho-verde) e nos casos mais graves apresentaram defeitos tritan-tetartan (azul-amarelo). Defeitos do tipo deutan e protan puderam ser encontrados em diversas distrofias, tais como na ritinite pigmentosa inversa e distrofia central areolar da coróide ${ }^{(7)}$. O teste de cores também pode ser normal, como acontece na distrofia macular da Carolina do Norte e na distrofia reticular do epitélio pigmentado da retina de Sjögren.

$\mathrm{Na}$ angiofluoresceinografia da DRORE, encontramos dispersões de tamanhos e formas variados e atrofia do EPR e da coriocapilar no pólo posterior; discos ópticos e vasos são normais. Esses achados são similares aos encontrados na distrofia central areolar da coróide, em que se encontra hiperfluorescência em área macular devido à atrofia do EPR e áreas de hipofluorescência com visualização dos grandes vasos e intermediários da coróide. De modo diferente da DRORE na distrofia central areolar da coróide, em que a alteração é restrita à área macular.

Na topografia de disco óptico (TOPSS), houve diminuição da rima neural no OD na paciente II-19. Houve a diminuição do diâmetro do disco óptico nos pacientes III-25 (sem distrofia macular) e III-21 (com distrofia macular); por acometer tanto o paciente normal como o afetado ocularmente, o diâmetro do disco óptico não está provavelmente relacionado à distrofia macular. Disco óptico pequeno pode, no entanto, estar relacionado com a neuropatia óptica isquêmica anterior não arterítica.
No CV, houve alteração típica do glaucoma na paciente II19. Nos pacientes (caso III-25 e III-20), sem alterações oculares o $\mathrm{CV}$ foi normal. Os outros pacientes apresentaram $\mathrm{CV}$ com alteração do $\mathrm{CV}$ central e com preservação do $\mathrm{CV}$ periférico (caso III-22) e com remanescente do CV central e preservação do CV periférico (caso III-24). Estas alterações estão presentes nas distrofias maculares, com alteração do CV central e preservação ou não do $\mathrm{CV}$ periférico, dependendo do grau de avanço da distrofia para a periferia da retina ${ }^{(7)}$.

Na parte II deste trabalho discutiremos o heredograma, as possíveis explicações para o comportamento paradoxal da DRORE, complementação da classificação dos tipos de ondas do ERG como um novo tipo de onda e possíveis locais de mutações dos genes para o seu rastreamento e identificação. É sugerida também a possibilidade de classificação da DRORE associada ao distúrbio capilar (SCAF), como uma nova entidade nosológica dentro das displasias ectodérmicas. Finalizando proporemos uma classificação desta nova entidade nosológica dentro do grupo B das displasias ectodérmicas.

\section{CONCLUSÃO}

Esta é a primeira descrição completa ao nosso conhecimento na literatura nacional de uma nova distrofia macular. São abordados a história, determinação da herança (vide parte II deste trabalho) e classificação quanto ao local de alterações na retina, através dos exames de eletrofisiologia ocular. O traçado eletrorretinográfico característico com tempo de culminação diminuído ou rápido na fase escotópica, pode ser considerado como fundamental para o diagnóstico dessa distrofia, cuja denominação sugerida é: Distrofia retiniana com onda rápida escotópica (DRORE) associada à síndrome dos cabelos anágenos frouxos (SCAF).

\section{ABSTRACT}

Purpose: To describe the ophthalmological findings of a new macular dystrophy associated with loose anagen hair syndrome (LAHS). Methods: Eleven patients of the same family, four of whom presented ocular abnormalities, were examined. Seven patients were submitted to the following examinations: a complete ophthalmological examination, color test, ultrasonography, angiography, visual field, optic disc topography, electro-oculogram (EOG), electroretinogram (ERG), laboratory and dermatological tests, sweat testing, light microscopy (LM) and scanning electron microscopy (SEM) of the scalp hair. In four patients, children of affected, examination was restricted to an ophthalmological examination and light microscopy and the scanning electron microscopy of the scalp hair. Results: Of the four affected patients, two affected sisters showed pigmentary dispersions in the posterior pole of the retina with macular coloboma. Two brothers showed pigmentary dispersions in the posterior pole, with greater pigmentation and a 
yellowish aspect of the macular area without coloboma. The results of the ectro-oculogram and electroretinogram were within normal limits in three patients without ocular abnormalities. Whereas, electro-oculogram was subnormal and electroretinogram was subnormal with short implicit time (fast) in the scotopic phase of the patients with the macular dystrophy, suggesting that the site of this dystrophy is at the level of the retinal pigment epithelium. Due to this peculiar behavior of the implicit time of the wave, such finding can be considered as fundamental to make the diagnosis of this dystrophy. The denomination could be scotopic fast wave retinal dystrophy (SFWRD). Light microscopy and scanning electron microscopy of scalp hair confirmed the loose anagen hair syndrome. Conclusions: We describe the findings of scotopic fast wave retinal dystrophy associated with the loose anagen hair syndrome, dystrophy whose fundoscopy findings differ between men and women and with characteristic electroretinographic tracing.

Keywords: Macular degeneration; Hair diseases, Syndrome; Coloboma; Microscopy, electron, scanning; Microscopy, electron; Electroretinography

\section{REFERÊNCIAS}

1. Deutman AF. The hereditary dystrophies of the posterior pole of the eye. Springfield, IL: Thomas; 1971.

2. Price VH, Gummer CL. Loose anagen syndrome. J Am Acad Dermatol 1989;20(2 Pt 1):249-56.

3. Baden HP, Kvedar JC, Magro CM. Loose anagen hair as a cause of hereditary hair loss in children. Arch Dermatol 1992;28:1349-53.

4. Sato MT. Distrofia retiniana com onda rápida escotópica (DRORE) associada à síndrome dos cabelos anágenos frouxos [tese]. Curitiba: Universidade Federal do Paraná; 2002.

5. Sato MT, Takahashi WY, Moreira Júnior CA. Influência da idade e da adaptação precoce ao claro, na normatização do eletrorretinograma de campo total em sujeitos normais. Rev Bras Oftalmol 2003;62:783-94.

6. Sato MT, Marzagão R, Graff C, Arana J, Moreira ATR, Pagnan NAB, et al. Descrição de nova distrofia macular associada à síndrome dos cabelos anágenos frouxos. Arq Bras Oftalmol 2002;65:249-56.

7. Cavender JC, Al E. Hereditary macular dystrophies. In: Tasman W, Jaeger EA, editors. Duane's Clinical Ophthalmology. Philadelphia: J.B Lippincott; 1990. p.1-29.

8. Kingham JD, Fenzl RE, Willerson D, Aaberg TM. Reticular dystrophy of the retinal pigment epithelium. Clinical and electrophysiologic study of three generations. Arch Ophthalmol 1978;96:1177-84

9. Crampton G, Armigton JC. Area-intensity relation and retinal location in the human electroretinogram. Am J Physiol 1955;181:47-53.

10. Berson EL, Gouras P, Hoff M. Temporal aspects of the electroretinogram. Arch Ophthalmol 1969;81:207-14.

11. Bornschein H, Goodman G, Gunkel RD. Temporal aspects of the human electroretinogram: a study of the implicit time-amplitude relationship of the B-wave. Arch Ophthalmol 1957;57:386-92.

12. Bornschein H, Goodman G. Studies of the a-wave in the human electroretinogram. Arch Ophthalmol 1957;58:431-7.

13. Brunette JR. The human electroretinogram during dark adaptation. Implicit time and amplitude studies. Arch Ophthalmol 1969;82:491-8.

14. Dorey CK, Wu G, Ebstein D, Garsd A, Witer JJ. Cell loss in the aging retina. Relationship to lipofuscin accumulation and macular degeneration. Invest Ophthalmol 1989;30:1691-9.

15. Tosti A, Colombati S, Padova MPD, Guidi SG, Tosti G, Maccolini E. Retinal pigment epithelium function in alopecia areata. J Invest Dermatol 1986;86:553-5.

16. Duke-Elder S, Dobree JHS. Degenerations and dystrophies. In: Duke-Elder S, editor. System of ophthalmology. Diseases of the retina. St. Louis: Mosby; 1967. p.591-3.

17. Goodman G, Ripps H, Siegel IM. Cone dysfunction syndromes. Arch Ophthalmol 1964;70:214-31.

18. Heckenlively JR, Foxman SG, Parelhoff ES. Retinal dystrophy and macular coloboma. Doc Ophthalmol 1988;68:257-71.

19. Murphy MF, McGinnity FG, Allen GE. New familial association between coloboma and loose anagen syndrome. Clin Genet 1995;47:214-6.

20. Harris GS, Yeung J. Maculophathy of sex-linked juvenile retinoschisis. Can J Ophthalmol 1976;11;1-10.

\section{CONGRESSO PAN-AMERICANO DE OFTALMOLOGIA}

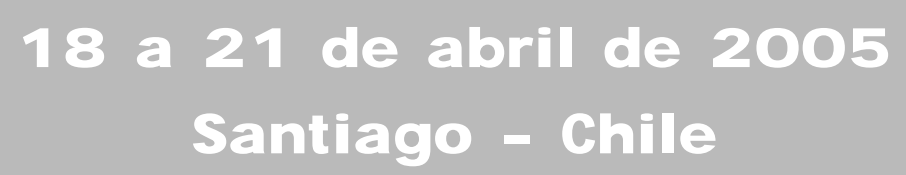

INFORMAÇÕES: e-mail: info@paao.org 